\begin{tabular}{|c|c|c|}
\hline & \multicolumn{2}{|c|}{ Journal of Applied Mathematics and Computational Mechanics 2020, 19(3), 29-43 } \\
\hline & www.amcm.pcz.pl & p-ISSN 2299-9965 \\
\hline & DOI: $10.17512 /$ jamcm.2020.3.03 & e-ISSN 2353-0588 \\
\hline
\end{tabular}

\title{
NUMERICAL SIMULATION OF CHANNEL FLOW OVER A SKEWED EQUILATERAL CAVITY
}

\author{
Abanoub G. Kamel, Eman H. Haraz, Sarwat N. Hanna \\ Department of Engineering Mathematics and Physics, Faculty of Engineering, Alexandria University \\ Alexandria 21544, Egypt \\ abanoub.george@alexu.edu.eg; eman.haraz@alexu.edu.eg; sarwat.hanna@alexu.edu.eg
}

Received: 6 April 2020; Accepted: 26 July 2020

\begin{abstract}
In this paper, an incompressible, two-dimensional (2D), time-dependent, Newtonian, laminar, and internal channel fluid flow over a skewed equilateral cavity is simulated using the finite difference method (FDM) and alternating direction implicit (ADI) technique. Navier-Stokes equations are solved numerically in stream function-vorticity formulation. The goal of tackling this problem depends on its academic significance by studying the difference between lid-driven and shear-driven cavity flows in terms of the formation of Moffatt eddies at the sharp corner, also to obtain the length and intensity ratios of these counter-rotating vortices. The value of velocity components along the centerlines of the skewed cavity was revealed at low and intermediate Reynolds numbers (Re), typically $(\mathrm{Re}=200$ and 2000$)$ at two different skew angles of mainly $30^{\circ}$ and $45^{\circ}$. Likewise, the blocked-off regions' method is used to deal with the geometry of the skewed cavity especially the sharp corners. Furthermore, as Re increases, the main vortex approaches the skewed cavity center and the counter-rotating vortices get bigger in size and intensity, and their number increases.
\end{abstract}

MSC 2010: 76D17, 76D05

Keywords: finite difference method, incompressible flow, channel flow, lid-driven, shear-driven, skewed cavity, Navier-Stokes equations

\section{Introduction}

The flow inside a two-dimensional (2D) lid-driven cavity was a motivating exploration field for many researchers as it is considered as a benchmark problem. Likewise, it is common because of its basic geometry and the significance of understanding the dynamics of primary as well as secondary generations of vortices and their location at the corners of the cavity where eddies are formed. Several numerical methods are examined by studying the 2D and 3D lid-driven cavity with different shapes and aspect ratios at low and very high Reynolds number (Re). The following articles are used by a plethora of researchers to verify their results 
(Ghia et al. [1], Gupta and Kalita [2], Ghadimi et al. [3], Zhang et al. [4], Ahmed and Kuhlmann [5], Abu-Nada and Chamkha [6], Botella and Peyret [7], Pinarbasi et al. [8], Wahba [9], and Kuhlmann and Romanò [10]). However, Romanò and Kuhlmann [11] studied the motion of suspended solid particles in a fluid for an extensive range of geometry and flow factors for many well-known problems using the smoothed-profile technique that was combined with high-order spatial discretization. The outcomes from their proposed method were accurate and efficient.

On the other hand, the orthogonal grid mesh will be used to solve channel flow over a skewed equilateral cavity using the finite difference method (FDM) and alternating direction implicit (ADI) scheme as stated by Peaceman and Rachford, $\mathrm{Jr}$ [12], besides a well-established technique called blocked-off regions' method to eliminate the unwanted region from the computational domain by setting the value of the variables at the cells which are located in the blocked regions to zero.

The benchmark problem of the flow inside a lid-driven skewed cavity was firstly presented by Demirdžić et al. [13]. He used the multigrid and finite volume methods on a non-orthogonal grid mesh and studied the fluid flow and heat transfer in two cases for investigating novel solution techniques. The skew angles in the two studied cases were $30^{\circ}$ and $45^{\circ}$.

Krishna et al. [14] examined in detail the impacts of Darcy number, Re, aspect ratio, porosity, and the skewness angle on the fluid flow in a lid-driven cavity filled with a permeable medium which was saturated with a liquid. They discovered that, with the increase in skew angle as well as the Re and with the decrease in Darcy number, the maximum stream function value decreased. Mohapatra [15] inspected the laminar and 2D fluid flow in a lid-driven skewed cavity. His results were obtained for a skew angle equal to $30^{\circ}$ and $45^{\circ}$ as well as Re values equal to 100 and 1000 .

Thohura et al. [16] numerically studied the case of Non-Newtonian laminar fluid flow in a lid-driven skewed cavity employing a power-law viscosity model. They took into their consideration the effects of the skew angle $\left(\alpha=15^{\circ}\right.$ to $\left.165^{\circ}\right)$ for both shear-thickening and shear-thinning fluids, the $\operatorname{Re}$ was $(\operatorname{Re}=100,200$, 300 , and 500) as well as the index of the power-law $(n=0.5,1$, and 1.5). Erturk and Dursun [17] revisited the fluid flow inside the lid-driven skewed cavity benchmark problem with more values of skew angles generally from $15^{\circ}$ to $165^{\circ}$. They utilized a numerical technique that was stable and very effective even at severe skew angles. Moreover, they obtained highly accurate results utilizing a fine grid typically (512 x 512). The results were found at low and high values of the Re specifically at 100 and 1000 .

Romanò and Kuhlmann [18] numerically studied the motion of a weighty tracer in a $2 \mathrm{D}$ shear-driven cavity. They used a smoothed profile technique along with a discontinuous Galerkin finite element approach. Nonetheless, they compared their results with those found by single coupling and found that the particles' true limit cycle could be approximated by the single coupling method using certain parameters like size and density. De Vicente et al. [19] linked between the centri- 
fugal instabilities' beginning and their last appearance in a fully saturated open cavity flow. They conducted a linear 3D modal instability analysis of steady 2D states that existed in an open cavity with an aspect ratio of 2 . Their results show that the structures of linear analysis are mostly compatible with the fully saturated experimental flow.

Moreover, the main cause of vortex generation inside the shear-driven skewed equilateral cavity will be the shear-force of the fluid layer above the cavity instead of a moving lid. The effect of the Reynolds number and skew angle on the large vortex as well as corner vortices generated at the sharp corner of the cavity and their length and intensity ratios as well as the centerline velocity profiles are found. The obtained results are compared to Moffatt eddies, and they were in a very good agreement. From the past review, it can be seen that the research purpose has not been investigated previously despite its academic value for the study of vortices' dynamics at sharp corners especially for the channel flow over a skewed equilateral cavity.

\section{Mathematical formulation}

\subsection{Problem statement}

Consider an incompressible, 2D, time-dependent, viscous, Newtonian, laminar, internal fluid flow in a channel over a skewed equilateral cavity. Figure 1 shows the dimensions of both the channel and the skewed cavity. The channel has an inlet height $\left(h_{i}\right)$ and the length from the beginning of the channel to the first tip of the skewed cavity $\left(L_{1}\right)$ and from the second tip of the skewed cavity to the end of the channel $\left(L_{2}\right)$, each will be taken equal to (3) times to the inlet height. Hence, the total length of the channel is equal to (6) times the inlet height and the length of the skewed cavity $(h)$. Moreover, the skew angle of the cavity with the horizontal is $(\theta)$. The selected values will be equal to one as follows; $h=h_{i}=1$ to be dimensionless.

In this problem, the non-dimensional Reynolds number can be described as $\operatorname{Re}=\frac{U D_{h}}{v}$ where $v$ is defined as the kinematic viscosity, $U$ is defined as the inlet mean velocity (it equals two-thirds of the maximum inlet velocity) and $D_{h}$ is defined as the hydraulic diameter of the channel inlet where it equals twice the inlet channel height, i.e. $D_{h}=2 h_{i}$.

In this study, the effect of Re, as well as the skew angle, will be revealed for the following values $\operatorname{Re}=200$ and 2000 , and $\theta=30^{\circ}$ and $45^{\circ}$. The shear-driven skewed cavity flow will have the main primary vortex besides secondary vortices at the acute and sharp corners. Furthermore, the flow at the inlet boundary has a parabolic velocity profile (fully developed flow) which is the Dirichlet type boundary condition (BC). However, the outlet velocity profile will also be parabolic according to the given dimensions. The value of the stream function $(\psi)$ and the vorticity $(\omega)$ can be determined by integrating and differentiating the $u$-velocity with respect to 
the $Y$-coordinate, respectively. The Exit flow has Neumann type BCs that are introduced for the three physical quantities: velocity, stream function, and vorticity. The no-slip velocity BC will be applied to all rigid walls, also the stream function values are prescribed while the vorticity values can be found using the Taylor series expansion with a truncation error from the first order also the inclined BCs can be found using the formulas described by Jenson [20] and used by Haese and Teubner [21].

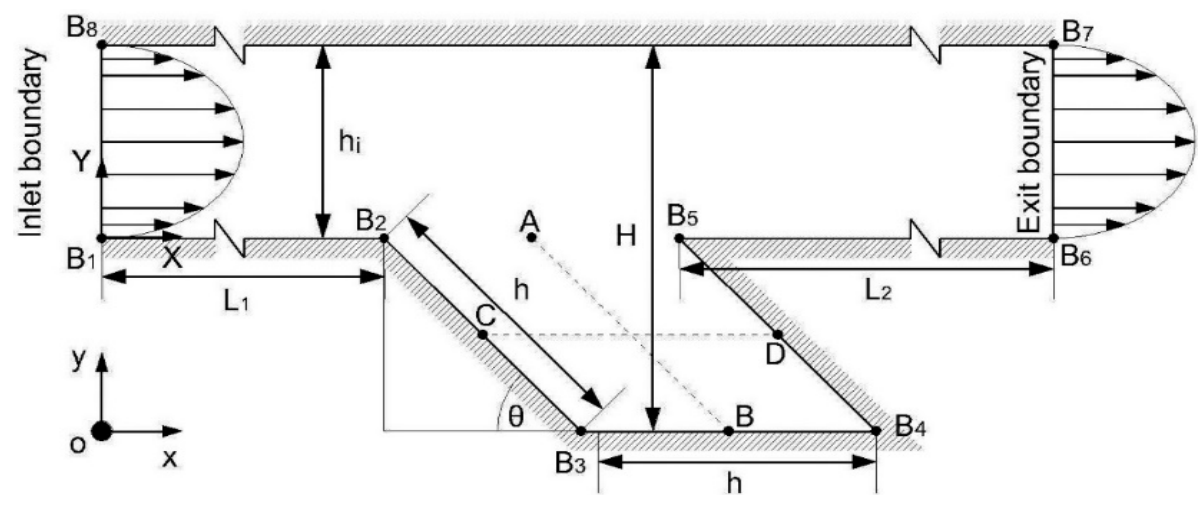

Fig. 1. Channel flow over a skewed equilateral cavity

\subsection{Governing equations}

The governing equations are the continuity Eq. (1) and the 2D Navier-Stokes Eq. (2) as well as Eq. (3) in Cartesian coordinates that are given as follows [22] and [23]:

$$
\begin{gathered}
\frac{\partial u}{\partial x}+\frac{\partial v}{\partial y}=0 \\
\frac{\partial u}{\partial t}+u \frac{\partial u}{\partial x}+v \frac{\partial u}{\partial y}=-\frac{1}{\rho} \frac{\partial p}{\partial x}+v\left(\frac{\partial^{2} u}{\partial x^{2}}+\frac{\partial^{2} u}{\partial y^{2}}\right)+g_{x} \\
\frac{\partial v}{\partial t}+u \frac{\partial v}{\partial x}+v \frac{\partial v}{\partial y}=-\frac{1}{\rho} \frac{\partial p}{\partial y}+v\left(\frac{\partial^{2} v}{\partial x^{2}}+\frac{\partial^{2} v}{\partial y^{2}}\right)+g_{y}
\end{gathered}
$$

where $\rho, v, u, v, p$, and $g$ are fluid density, kinematic viscosity, velocity components along $x$ and $y$ axes, pressure, and gravitational acceleration, respectively.

For a two-dimensional flow, the vorticity $(\omega)$ at a certain fluid point is given by

$$
\omega=\frac{\partial v}{\partial x}-\frac{\partial u}{\partial y}
$$


Defining the stream function $(\psi)$ in Cartesian coordinate through

$$
u=\frac{\partial \psi}{\partial y}, v=-\frac{\partial \psi}{\partial x}
$$

The dimensionless differential form of the parabolic vorticity transport Eq. (6) is

$$
\frac{\partial \omega}{\partial t}+\frac{\partial \psi}{\partial y} \frac{\partial \omega}{\partial x}-\frac{\partial \psi}{\partial x} \frac{\partial \omega}{\partial y}=\frac{1}{\operatorname{Re}}\left(\frac{\partial^{2} \omega}{\partial x^{2}}+\frac{\partial^{2} \omega}{\partial y^{2}}\right)
$$

Now, after the substitution of Eq. (5) in Eq. (4), the final dimensionless differential form of the elliptic stream function equation, known as Poisson equation (7), is

$$
\frac{\partial^{2} \psi}{\partial x^{2}}+\frac{\partial^{2} \psi}{\partial y^{2}}=-\omega
$$

Once the stream function has been computed, the velocity components can be found using Eq. (5). The complete ADI method is described by Kamel et al. [24].

\section{Numerical discretization and boundary conditions}

To discretize the computational domain, a structured collocated grid will be chosen for this problem where the value of any variable $(\omega, \psi, u$, and $v)$ will be stored at the same point and at two different time steps on a five-point stencil. Also, a forward difference for time discretization and central difference for spatial discretization known as (FTCS) will be applied for the first and second partial derivatives in Eqs. (6) and (7). The resulting accuracy from using the ADI method along with the FDM will be from the second-order in time and space $\mathrm{O}\left((\Delta t)^{2}\right.$, $(\Delta x)^{2}$ and $\left.(\Delta y)^{2}\right)$ and it is unconditionally stable according to Hoffmann and Chiang [22]. Moreover, the benefit of using the stream function-vorticity form is the satisfaction of the continuity Eq. (1). By solving the vorticity transport Eq. (6), the vorticity value can be obtained. The vorticity values will then be introduced to the Poisson Eq. (7). The inflow, outflow, and solid BCs are introduced in Table (1) for all variables $(u, v, \psi$, and $\omega)$.

Where the subscript (0) denotes for the points on the rigid inclined wall and the subscripts (11) and (12) denote for the points adjacent to the rigid inclined wall in the $y$-direction and the $x$-direction, respectively. 
Table 1. Boundary conditions for channel flow over a skewed equilateral cavity

\begin{tabular}{|c|c|c|c|}
\hline \multirow{2}{*}{$\begin{array}{l}\text { Boundary } \\
\text { code and type }\end{array}$} & \multicolumn{3}{|c|}{ Physical quantity } \\
\hline & Velocity $(u, v)$ & Stream function $(\psi)$ & Vorticity $(\omega)$ \\
\hline B1B8 (Inlet) & $\begin{array}{c}u=6 *\left(Y-Y^{2}\right) \\
v=0\end{array}$ & $\psi=-2 * Y^{3}+3 * Y^{2}$ & $\omega=-\frac{\partial u}{\partial y}=12 * Y-6$ \\
\hline B6B7 (Exit) & $\frac{\partial u}{\partial x}=0, \frac{\partial v}{\partial y}=0$ & $\frac{\partial \psi}{\partial x}=0$ & $\frac{\partial \omega}{\partial x}=0$ \\
\hline B7B8 (Top) & $\begin{aligned} u & =0 \\
v & =0\end{aligned}$ & $\psi=-2 * h_{i}{ }^{3}+3 * h_{i}{ }^{2}$ & $\omega_{0}=-\frac{\partial^{2} \psi}{\partial y^{2}}=\frac{2\left(\psi_{0}-\psi_{11}\right)}{(\Delta y)^{2}}$ \\
\hline $\begin{array}{c}\text { B1B2, B3B4, } \\
\text { B5B6 } \\
\text { (Bottom) }\end{array}$ & $\begin{array}{l}u=0 \\
v=0\end{array}$ & $\psi=0$ & $\omega_{0}=-\frac{\partial^{2} \psi}{\partial y^{2}}=\frac{2\left(\psi_{0}-\psi_{11}\right)}{(\Delta y)^{2}}$ \\
\hline $\begin{array}{l}\text { B2B3, B4B5 } \\
\text { (Inclined) }\end{array}$ & $\begin{array}{l}u=0 \\
v=0\end{array}$ & $\psi=0$ & $\begin{array}{c}\omega_{0}=-\frac{\partial^{2} \psi}{\partial x^{2}}-\frac{\partial^{2} \psi}{\partial y^{2}}= \\
=\frac{2\left(\psi_{0}-\psi_{11}\right)}{(\Delta y)^{2}}+\frac{2\left(\psi_{0}-\psi_{12}\right)}{(\Delta x)^{2}}\end{array}$ \\
\hline
\end{tabular}

\section{Numerical computations}

The numerical computation of the flow variables $(u, v, \psi$, and $\omega)$ have been obtained for the current problem with the assistance of a computer software code which has been verified and validated by studying the one-sided lid-driven square cavity problem by Kamel et al. [24]. The solution convergence for each Re was measured by the residual of the vorticity Eq. (8) as indicated in [3], which is given as:

$$
R_{\omega}=\sum_{i=2, j=2}^{i=n x, j=n y}\left(\omega_{i, j}^{n+1}-\omega_{i, j}^{n}\right)^{2} .
$$

where $R_{\omega}$ is the vorticity residual and $n$ is the number of iterations and the convergence criterion is set to $\left(10^{-10}\right)$ for each Re. Likewise, grid independence study for current work at $\left(\operatorname{Re}=200\right.$ and $\left.\theta=45^{\circ}\right)$ is shown in Table 2 and it is obvious that a grid of $(466 \times 115)$ produces a small difference in the stream function value compared to the grid of $(239 \times 59)$ while it is almost double the grid size. Therefore, the $(466 \times 115)$ grid is enough for the simulation, and in the event of a different Re or skew angle, a grid study was done in the same manner. 
Table 2. Stream function and vorticity values and the main vortex center at $\mathrm{Re}=200$ and $\theta=45^{\circ}$

\begin{tabular}{|c|c|c|c|c|c|c|}
\hline Grid size & $\Delta x$ & $\Delta y$ & $\psi_{\min }$ & $\omega_{\text {v.c. }}$ & $x$ & $y$ \\
\hline $70 \times 18$ & 0.1 & 0.1 & -0.0239 & -1.1666 & 3.8551 & 0.5021 \\
\hline $90 \times 23$ & 0.08 & 0.08 & -0.0223 & -1.0733 & 3.8539 & 0.4656 \\
\hline $120 \times 30$ & 0.06 & 0.06 & -0.0193 & -1.2831 & 3.8235 & 0.5298 \\
\hline $179 \times 45$ & 0.04 & 0.04 & -0.0178 & -1.0689 & 3.7753 & 0.5044 \\
\hline $239 \times 59$ & 0.03 & 0.03 & -0.0173 & -1.0284 & 3.7647 & 0.5004 \\
\hline $466 \times 115$ & 0.015 & 0.015 & -0.0167 & -1.0012 & 3.7785 & 0.4942 \\
\hline
\end{tabular}

\section{Numerical results}

In the case when the skew angle is $30^{\circ}$, Figure 2 shows the streamlines of the large primary vortex that dominate the skewed cavity and also presents the smaller secondary vortices which are generated at the sharp corner. Additionally, as Re increases, the primary vortex forms a line that is approximately horizontal and connects the two tips of the skewed cavity. Figure 3 illustrates the vorticity contours which in turn represent the location of the main vortex and the smaller corner eddies. As Re increases, the vorticity contours start to take the shape of the large vortex while the contours of the smaller secondary vortices did not seem to change in their shape. However, the intensity and size of the smaller secondary vortices increased which is indicated by the vorticity contours concentration.

(a)

(y)

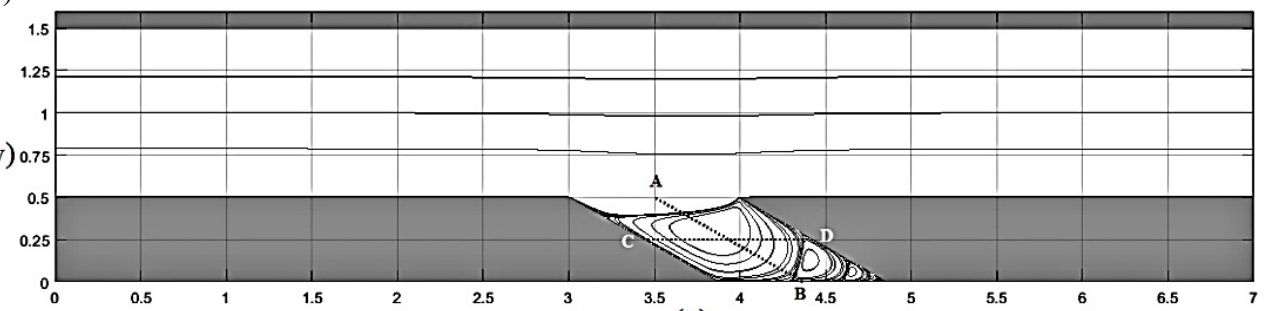

(x)

(b)

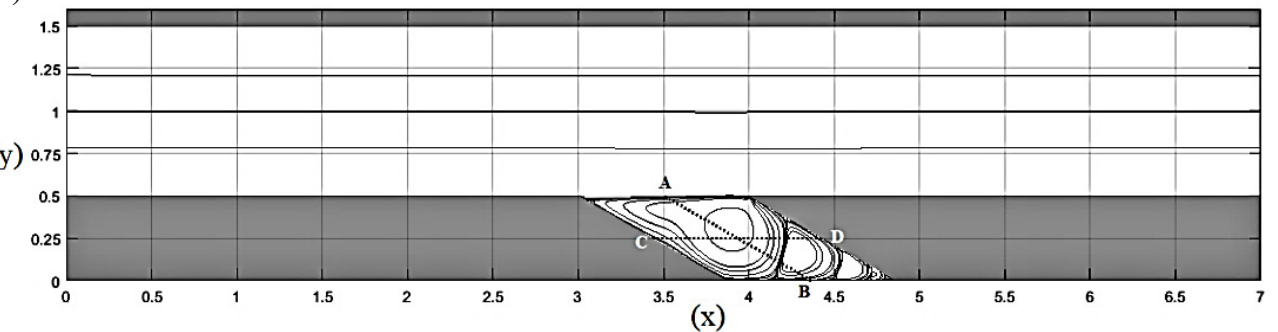

Fig. 2. Streamlines for $\operatorname{Re}(a) 200$ and (b) 2000 and skew angle is $30^{\circ}$ 
(a)

(y)
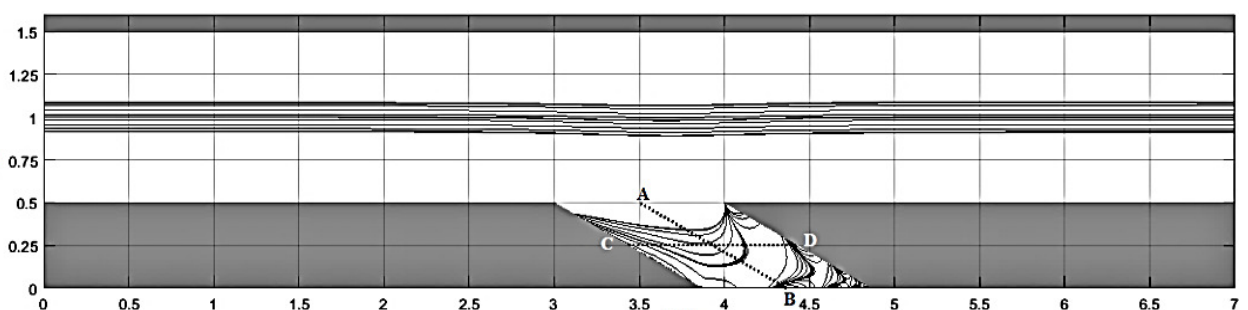

(x)

(b)

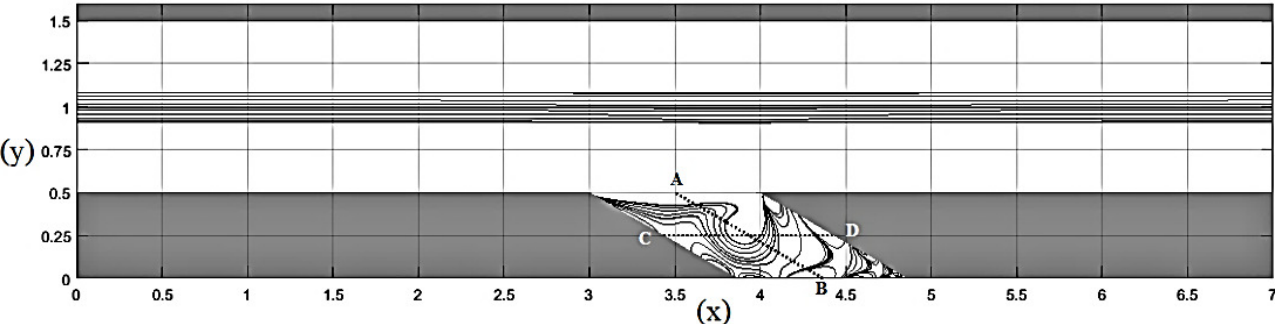

Fig. 3. Vorticity contours for Re (a) 200 and (b) 2000 and skew angle is $30^{\circ}$

It is detected from Figure 4 that, as the Re increases, the maximum value of the centerline $u$-velocity along A-B at the top of the skewed cavity decreases with more wiggles in the profile and the cause of the velocity reduction may be due to point (A) located at the top of the cavity entered the large vortex (instead of remaining in the freestream) where energy loss occurred. However, the difference between peaks and bottoms in the profiles of the centerline $v$-velocity along C-D increases.

(a)

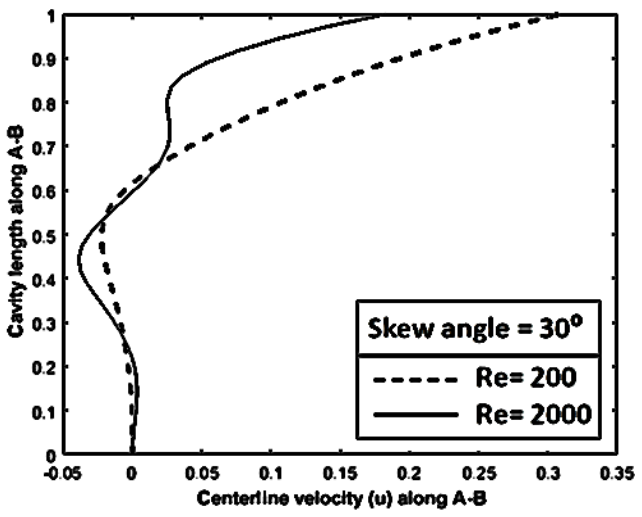

(b)

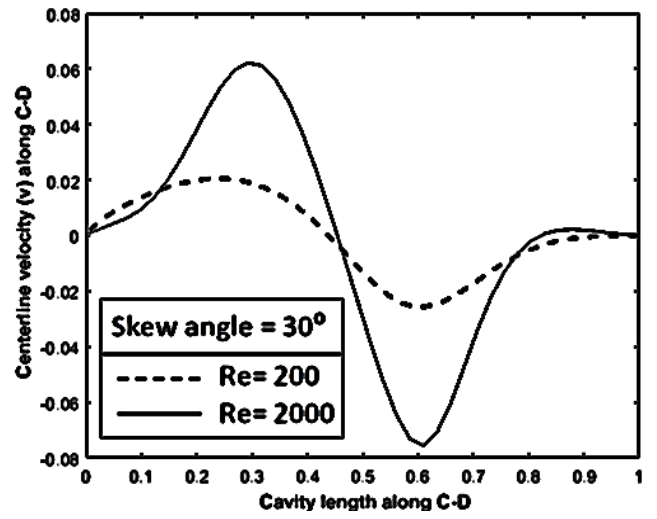

Fig. 4. Centerline velocity profiles for channel flow over a skewed equilateral cavity (a) $u$, along A-B and (b) $v$, along C-D and skew angle is $30^{\circ}$

Figure 5 shows the difference between the velocity profiles of the channel flow at the inlet as well as the exit boundaries of the channel at $\mathrm{Re}=200$ and 2000 . 
Both of them at a skew angle of $30^{\circ}$. This demonstrates that the exit velocity profile is parabolic and nearly the same as the inlet velocity profile.

(a)

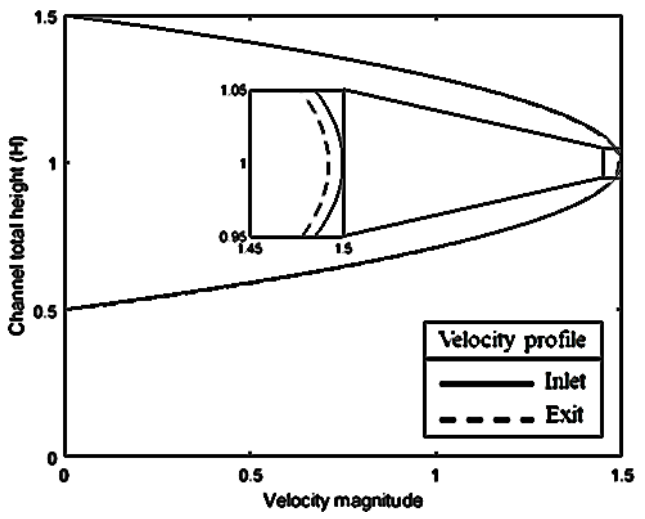

(b)

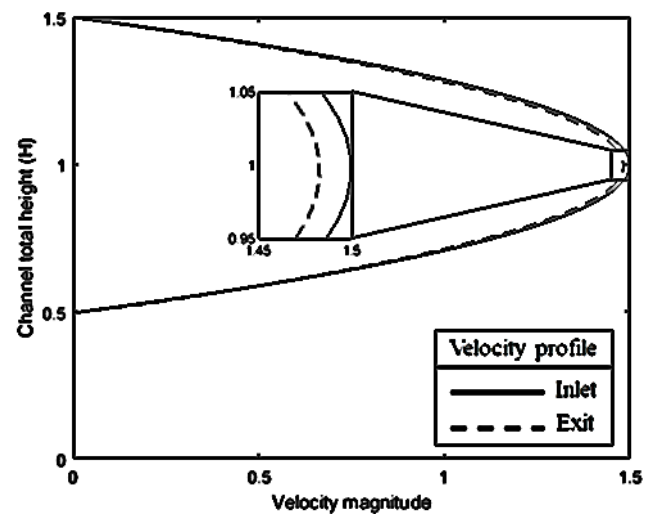

Fig. 5. Velocity profiles at the channel inlet and exit for Re (a) 200 and (b) 2000 and skew angle is $30^{\circ}$

It is noteworthy to say that the Re of 200 and 2000 in the current work (sheardriven cavity) is comparable to Re of 100 and 1000 in the lid-driven cavity problem because, in this problem, the non-dimensional Reynolds number is based on the hydraulic diameter $\left(D_{h}\right)$ of the channel inlet where it equals twice the inlet channel height, i.e. $D_{h}=2 h_{i}$. Also, Table 3 illustrates that as the Re increases the $\left|\psi_{\min }\right|$ as well as $\psi_{\max }$ values and their corresponding $\left|\omega_{v . c .}\right|$ values also increase for present and Erturk and Dursun [17] results except for the values of $\left|\omega_{\text {v.c. }}\right|$ corresponding to $\left|\psi_{\min }\right|$ that decreased as indicated by Erturk and Dursun [17]. Moreover, the ( $x$-locations) of the vortices appears inside parentheses are measured from the beginning of the very bottom left corner of the skewed cavity for a fair comparison.

Table 3. $\psi_{\min }, \psi_{\max }$, and $\omega_{v . c .}$ values and location of vortices' centers at $30^{\circ}$

\begin{tabular}{|c|c|c|c|c|}
\hline \multirow{2}{*}{ Property } & \multicolumn{2}{|c|}{ Present at $\theta=30^{\circ}$} & \multicolumn{2}{c|}{ Erturk and Dursun [17] at $\theta=150^{\circ}$} \\
\cline { 2 - 5 } & $\mathrm{Re}=200$ & $\mathrm{Re}=2000$ & $\mathrm{Re}=100$ & $\mathrm{Re}=1000$ \\
\hline$\psi_{\min }$ & -0.0072 & -0.0157 & -0.062347 & -0.071681 \\
\hline$\omega_{\text {v.c. }}$ & -0.8544 & -1.3646 & -5.46336 & -4.35978 \\
\hline$x$ & $3.8806(0.0146)$ & $3.8834(0.0174)$ & $(-0.0074)$ & $(0.0478)$ \\
\hline$y$ & 0.3190 & 0.2972 & 0.3516 & 0.2803 \\
\hline$\psi_{\max }$ & $7.9896 \mathrm{E}-06$ & $3.6487 \mathrm{E}-04$ & $1.0998 \mathrm{E}-04$ & $3.4432 \mathrm{E}-03$ \\
\hline$\omega_{\text {v.c. }}$ & 0.0051 & 0.1205 & 0.060074 & 1.32398 \\
\hline$x$ & $4.4030(0.5369)$ & $4.2792(0.4131)$ & $(0.5003)$ & $(0.3994)$ \\
\hline$y$ & 0.1293 & 0.1557 & 0.1396 & 0.1348 \\
\hline
\end{tabular}


The $\left|\psi_{\min }\right|$ as well as $\psi_{\max }$ values and their corresponding $\left|\omega_{v . c .}\right|$ values are greater in the case of a lid-driven (Erturk and Dursun [17]) than a shear-driven (present) skewed cavity because the lid-driven cavity is a confined space where there is no other space for the vortices to extend or stretch. On the contrary, in the current study, the vortices are left to stretch or extend freely.

The intensity ratio of the corner eddies is verified by comparison with Erturk and Dursun [17] and Moffatt [25] as shown in Table 4. The present ratio at $\left(\operatorname{Re}=200 \& \theta=30^{\circ}\right)$ is higher than that of Erturk and Dursun [17] at $(\operatorname{Re}=100$ $\& \theta=150^{\circ}$ ) but both are higher than that of Moffatt [25] at $2 \alpha=30^{\circ}$ at low Re. Nonetheless, the present ratio at $\left(\operatorname{Re}=2000 \& \theta=30^{\circ}\right)$ is higher than that of Erturk and Dursun [17] at $\left(\operatorname{Re}=1000 \& \theta=150^{\circ}\right)$ and are almost half the value of Moffatt [25] at $2 \alpha=30^{\circ}$.

Table 4. Comparison of the ratios of intensities of successive sharp corner eddies at $30^{\circ}$

\begin{tabular}{|c|c|c|c|c|}
\hline \multicolumn{4}{|c|}{ The ratios of intensities of successive sharp corner eddies $\left|\psi_{\min }\right| / \psi_{\max }$ and $\left(\ln \left(\left|\psi_{\min }\right| / \psi_{\max }\right)\right)$} \\
\hline \multicolumn{4}{|c|}{ Present at $\theta=30^{\circ}$} & \multicolumn{3}{|c|}{ Erturk and Dursun [17] at $\theta=150^{\circ}$} & Moffatt [25] at $2 \alpha=30^{\circ}$ \\
\hline $\mathrm{Re}=200$ & $\mathrm{Re}=2000$ & $\mathrm{Re}=100$ & $\operatorname{Re}=1000$ & Low $\operatorname{Re}$ \\
\hline $901.17(6.80)$ & $43.03(3.76)$ & $566.89(6.34)$ & $20.82(3.04)$ & $411.58(6.02)$ \\
\hline
\end{tabular}

In the second case when the skew angle is $45^{\circ}$, Figure 6 is similar to Figure 2, nonetheless they have some major differences such as the primary vortex is larger and the number of the smaller eddies is lesser when the skew angle is $45^{\circ}$ than $30^{\circ}$.

(a)

(y)

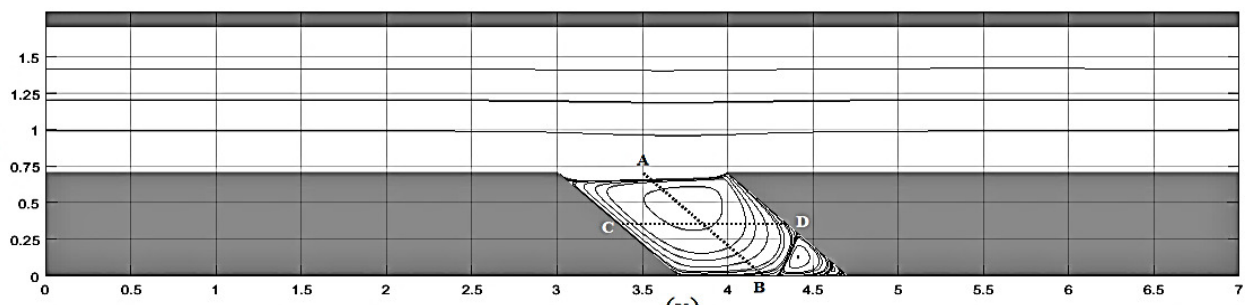

(b)

(x)

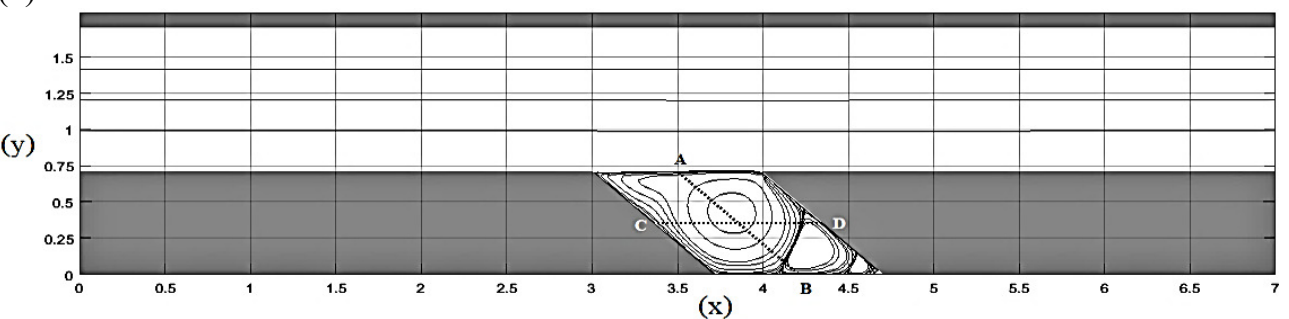

Fig. 6. Streamlines for Re (a) 200 and (b) 2000 and skew angle is $45^{\circ}$

Figure 7 illustrates the vorticity contours which in turn represent the location of the main vortex and the smaller corner eddies. Moreover, as Re increases, the vor- 
ticity contours start to take the shape of the large vortex while the contours of the smaller secondary vortices did not seem to change in their shape as in the earlier case. Furthermore, their intensity and size increased with more generated smaller eddies, as indicated by the concentration of the vorticity contours, as Re soared.

It is noteworthy to say that the number of the sharp corner eddies when the skew angle is equal to $30^{\circ}$ is higher than that when the skew angle is equal to $45^{\circ}$, and it is verified also by Erturk and Dursun [17].

(a)

(y)

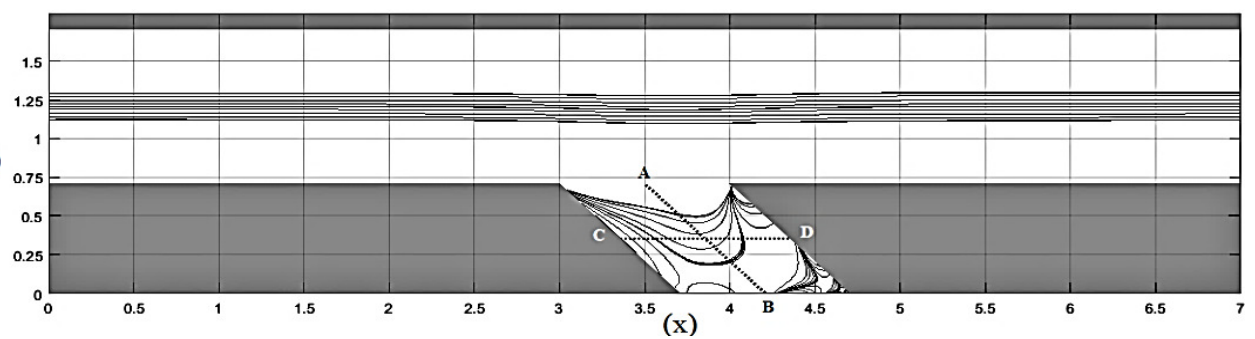

(b)

(y)

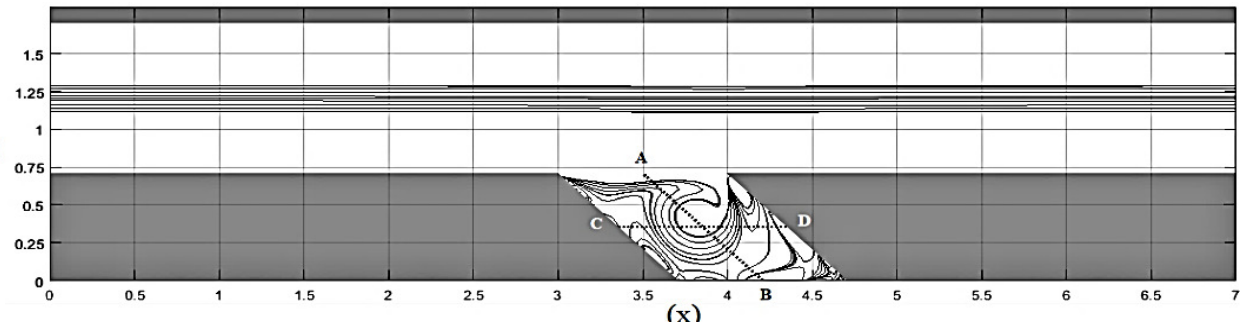

Fig. 7. Vorticity contours for Re (a) 200 and (b) 2000 and skew angle is $45^{\circ}$

(a)

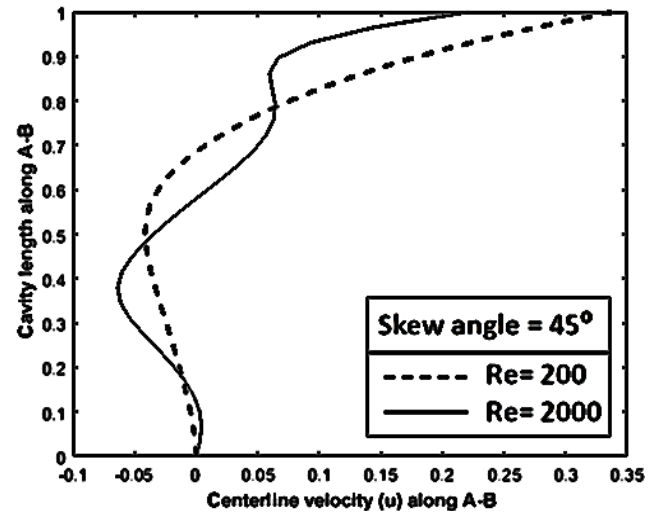

(b)

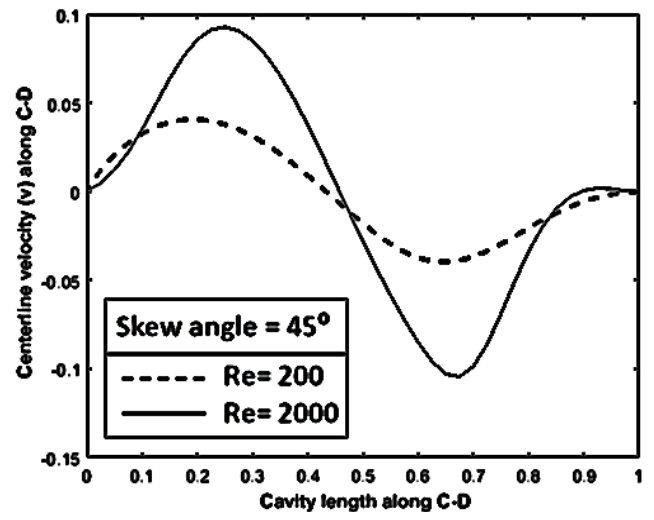

Fig. 8. Centerline velocity profiles for channel flow over a skewed equilateral cavity (a) $u$, along A-B and (b) $v$, along C-D and skew angle is $45^{\circ}$

Figure 8 shows that as the Re increases, the maximum value of the centerline $u$-velocity along A-B at the top of the skewed cavity decreases with more wiggles 
in the profile and the cause of the velocity reduction is the same as that when $\theta=30^{\circ}$. While the difference between peaks and bottoms in the profiles of the centerline $v$-velocity along C-D increases.

Figure 9 shows the difference between the velocity profiles of the channel flow at the inlet as well as the exit boundaries of the channel at $R e=200$ and 2000. Both of them are at a skew angle of $45^{\circ}$. This demonstrates that the exit velocity profile is parabolic and nearly the same as the inlet velocity profile.

(a)

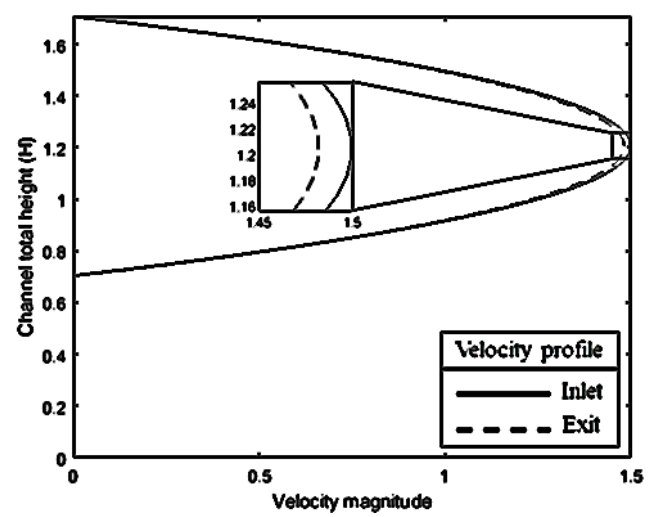

(b)

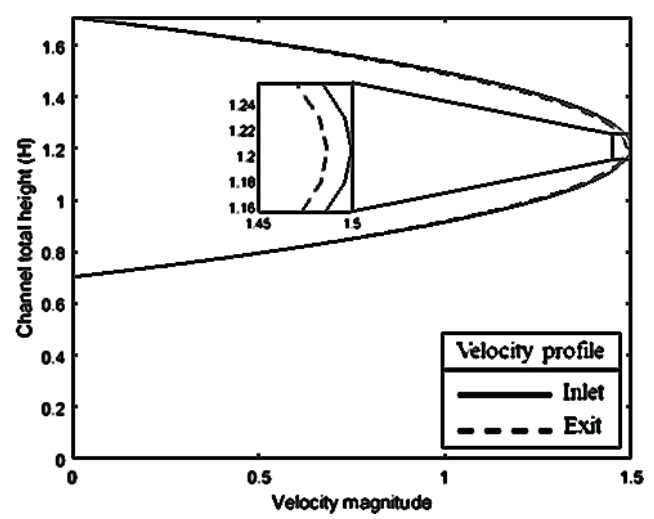

Fig. 9. Velocity profiles at the channel inlet and exit for Re (a) 200 and (b) 2000 and skew angle is $45^{\circ}$

It can be seen from Table (5) that, as the Re increases the $\left|\psi_{\min }\right|$ as well as $\psi_{\max }$ values and their corresponding $\left|\omega_{v . c .}\right|$ values also increase for present and Erturk and Dursun [17] results except for the values of $\left|\omega_{\text {v.c. }}\right|$ corresponding to $\left|\psi_{\text {min }}\right|$ that decreased as indicated by Erturk and Dursun [17].

The $\left|\psi_{\min }\right|$ as well as $\psi_{\max }$ values and their corresponding $\left|\omega_{\text {v.c. }}\right|$ values are greater in the case of a lid-driven (Erturk and Dursun [17]) than a shear-driven (present) skewed cavity and the cause of that surge is the same as that when $\theta=30^{\circ}$.

The most important thing from the data tabulated in Table 3 and Table 5 is that the present $\left|\psi_{\min }\right|, \psi_{\max }$ and their corresponding $\left|\omega_{\text {v.c. }}\right|$ values at both $(\operatorname{Re}=200$ and 2000) are greater when the skew angle is $45^{\circ}$ than those when the skew angle is $30^{\circ}$ except in the case when the skew angle is $30^{\circ}$ and for $\operatorname{Re}=2000$ the $\left|\omega_{\text {v.c. }}\right|$ value is greater than its value when the skew angle is $45^{\circ}$.

In the same way, the intensity ratio of the corner eddies is verified by comparison with Erturk and Dursun [17] and Moffatt [25] as shown in Table 6. The present ratio at $\mathrm{Re}=200 \& \theta=45^{\circ}$ is higher than that of Erturk and Dursun [17] at $\operatorname{Re}=100 \& \theta=135^{\circ}$ but both are higher than that of Moffatt [25] at $2 \alpha=45^{\circ}$ at low Re. Furthermore, the present ratio at $\operatorname{Re}=2000 \& \theta=45^{\circ}$ is also higher than that of Erturk and Dursun [17] at $\operatorname{Re}=1000 \& \theta=135^{\circ}$ and are almost half the value of Moffatt [25] at $2 \alpha=45^{\circ}$ at low Re. 
Table 5. $\psi_{\text {min }}, \psi_{\max }$, and $\omega_{\text {v.c. }}$ values and location of vortices' centers at $45^{\circ}$

\begin{tabular}{|c|c|c|c|c|}
\hline \multirow{2}{*}{ Property } & \multicolumn{2}{|c|}{ Present at $\theta=45^{\circ}$} & \multicolumn{2}{c|}{ Erturk and Dursun [17] at $\theta=135^{\circ}$} \\
\cline { 2 - 5 } & $\mathrm{Re}=200$ & $\mathrm{Re}=2000$ & $\mathrm{Re}=100$ & $\mathrm{Re}=1000$ \\
\hline$\psi_{\min }$ & -0.0167 & -0.0262 & -0.083704 & -0.093512 \\
\hline$\omega_{\text {v.c. }}$ & -1.0012 & -1.3393 & -4.06484 & -2.99180 \\
\hline$x$ & $3.7785(0.0714)$ & $3.8159(0.1088)$ & 0.1055 & 0.1390 \\
\hline$y$ & 0.4942 & 0.4268 & 0.4999 & 0.3922 \\
\hline$\psi_{\max }$ & $1.0132 \mathrm{E}-05$ & $5.8586 \mathrm{E}-04$ & $1.0764 \mathrm{E}-04$ & $3.7805 \mathrm{E}-03$ \\
\hline$\omega_{\text {v.c. }}$ & 0.0065 & 0.1831 & 0.057419 & 1.19738 \\
\hline$x$ & $4.4108(0.7037)$ & $4.2455(0.5384)$ & 0.6708 & 0.5554 \\
\hline$y$ & 0.1198 & 0.1757 & 0.1436 & 0.1478 \\
\hline
\end{tabular}

Table 6. Comparison of the ratios of intensities of successive sharp corner eddies at $45^{\circ}$

The ratios of intensities of successive sharp corner eddies $\left|\psi_{\min }\right| / \psi_{\max }$ and $\left(\ln \left(\left|\psi_{\min }\right| / \psi_{\max }\right)\right)$

\begin{tabular}{|c|c|c|c|c|}
\hline \multicolumn{2}{|c|}{ Present at $\theta=45^{\circ}$} & \multicolumn{2}{c|}{ Erturk and Dursun [17] at $\theta=135^{\circ}$} & Moffatt [25] at $2 \alpha=45^{\circ}$ \\
\hline $\mathrm{Re}=200$ & $\mathrm{Re}=2000$ & $\mathrm{Re}=100$ & $\mathrm{Re}=1000$ & Low $\mathrm{Re}$ \\
\hline $1648.24(7.41)$ & $44.72(3.80)$ & $777.63(6.66)$ & $24.74(3.21)$ & $518.01(6.25)$ \\
\hline
\end{tabular}

On the other hand, the data tabulated in Table 7 reveals the natural logarithm of the asymptotic length ratios of the corner eddies, and they are verified by comparison with Moffatt [25]. We can see that our results agree very well with Moffatt [25] for both the Re at 200 and 2000 and the skew angle when $\theta=2 \alpha=30^{\circ}$ and $45^{\circ}$.

Table 7. Comparison of the length ratios of successive sharp corner eddies at $30^{\circ}$ and $45^{\circ}$

\begin{tabular}{|c|c|c|c|c|c|}
\hline \multicolumn{6}{|c|}{ The natural logarithm (ln) of the length ratios of successive sharp corner eddies } \\
\hline \multicolumn{3}{|c|}{$\theta=2 \alpha=30^{\circ}$} & \multicolumn{3}{|c|}{$\theta=2 \alpha=45^{\circ}$} \\
\hline \multicolumn{2}{|c|}{ Present } & \multirow{2}{*}{$\begin{array}{c}\text { Moffatt [25] } \\
\text { Low Re }\end{array}$} & \multicolumn{2}{|c|}{ Present } & Moffatt [25] \\
\hline $\operatorname{Re}=200$ & $\mathrm{Re}=2000$ & & $\mathrm{Re}=200$ & $\mathrm{Re}=2000$ & Low $\mathrm{Re}$ \\
\hline 0.75 & 0.73 & 0.75 & 1.18 & 1.10 & 1.15 \\
\hline
\end{tabular}

\section{Conclusions}

It is noteworthy to say that, to our best knowledge, there is not a single investigation of channel flow over a skewed equilateral cavity. In addition, it has high academic value for the study of vortices dynamics at corners which depends on the Re, the number, and the direction of moving walls, and the skewness angle. 
Nonetheless, the introduced results of the present investigation are unique so, for future references, these outcomes offer a vital source for researchers to verify their outcomes. The following concluding remarks are based on the data described in the previous figures and tables that can be summarized as follows:

- As Re increases, the main vortex approaches the center of the skewed cavity.

- Likewise, the intensity, as well as the size of the smaller secondary vortices, increase. Also, at the lower sharp corner, there shows up continuously smaller counter-rotating recirculating eddies at high $\mathrm{Re}$ and most of them are generated at the fourth quad of the skewed cavity for both angles $30^{\circ}$ and $45^{\circ}$.

- Additionally, the higher the Re, the bigger the main vortex and it forms an approximately horizontal line and connecting the two tips of the skewed cavity for both skew angles $30^{\circ}$ and $45^{\circ}$.

- For a specific Re, as the skew angle decreases, the more counter-rotating vortices are generated at the sharp corner of the cavity.

- Surprisingly, in the presented shear-driven skewed cavity problem at a high Reynold number, there is no tertiary vortex generated at the left inclined wall of the skewed equilateral cavity at both skew angles $30^{\circ}$ and $45^{\circ}$ as the case in the lid-driven skewed cavity benchmark problem.

- The maximum value of the centerline $u$-velocity along A-B is not equal to one as the case in a lid-driven skewed cavity, because the moving lid is replaced with a moving shear layer of the flowing fluid.

- It is observed that the maximum values of the centerline u-velocity profile along A-B at the top of the skewed cavity when $\theta=45^{\circ}$ are greater than those when $\theta=30^{\circ}$. Similarly, the maximum, as well as the absolute of the minimum values of the centerline v-velocity profile along $\mathrm{C}$-D in the middle of the skewed cavity when $\theta=45^{\circ}$, are greater than those when $\theta=30^{\circ}$.

- The intensity and logarithm of the asymptotic length ratios of the corner eddies increase as the skew angle increases and they have been compared with Moffatt, and they were in a very good agreement.

The extension of the present work is to study the effect of changing the aspect ratio of the skewed cavity, the flow of Non-Newtonian fluid, turbulent flow, and the heat transfer on fluid flow. Also, the effect of the entrance region and channel height can be examined at a later point.

\section{References}

[1] Ghia, U., Ghia, K.N., \& Shin, C. (1982). High-Re solutions for incompressible flow using the Navier-Stokes equations and a multigrid method. Journal of Computational Physics, 48(3), 387-411.

[2] Gupta, M.M., \& Kalita, J.C. (2005). A new paradigm for solving Navier-Stokes equations: streamfunction - velocity formulation. Journal of Computational Physics, 207(1), 52-68.

[3] Ghadimi, P., Fard, M.Y., \& Dashtimanesh, A. (2013). Application of an Iterative high order difference scheme along with an explicit system solver for solution of stream function-vorticity form of Navier-Stokes equations. Journal of Fluids Engineering, 135(4), 1401. 
[4] Zhang, T., Shi, B., \& Chai, Z. (2010). Lattice Boltzmann simulation of lid-driven flow in trapezoidal cavities. Computers \& Fluids, 39(10), 1977-1989.

[5] Ahmed, M., \& Kuhlmann, H.C. (2012). Flow instability in triangular lid-driven cavities with wall motion away from a rectangular corner. Fluid Dynamics Research, 44(2), 5501.

[6] Abu-Nada, E., \& Chamkha, A.J. (2010). Mixed convection flow in a lid-driven inclined square enclosure filled with a nanofluid. European Journal of Mechanics-B/Fluids, 29(6), 472-482.

[7] Botella, O., \& Peyret, R. (1998). Benchmark spectral results on the lid-driven cavity flow. Computers \& Fluids, 27(4), 421-433.

[8] Ozalp, C., Pinarbasi, A., \& Sahin, B. (2010). Experimental measurement of flow past cavities of different shapes. Experimental Thermal and Fluid Science, 34(5), 505-515.

[9] Wahba, E. (2012). Steady flow simulations inside a driven cavity up to Reynolds number 35,000. Computers \& Fluids, 66, 85-97.

[10] Kuhlmann, H.C., \& Romanò, F. (2019). The lid-driven cavity. Computational Modelling of Bifurcations and Instabilities in Fluid Dynamics. Springer, 233-309.

[11] Romano, F., \& Kuhlmann, H.C. (2017). Smoothed-profile method for momentum and heat transfer in particulate flows. International Journal for Numerical Methods in Fluids, 83(6), 485-512.

[12] Peaceman, D.W., Rachford, J., \& Henry H. (1955). The numerical solution of parabolic and elliptic differential equations. Journal of the Society for Industrial and Applied Mathematics, $3(1), 28-41$.

[13] Demirdžić, I., Lilek, Ž., \& Perić, M. (1992). Fluid flow and heat transfer test problems for non-orthogonal grids: bench-mark solutions. International Journal for Numerical Methods in Fluids, 15(3), 329-354.

[14] Jaya Krishna, D., Basak, T., \& Das, S.K. (2008). Numerical study of lid-driven flow in orthogonal and skewed porous cavity. Communications in Numerical Methods in Engineering, 24(10), 815-831.

[15] Mohapatra, R.C. (2016). Study on laminar two-dimensional lid-driven cavity flow with inclined side wall. Open Access Library Journal, 3(03), 1.

[16] Thohura, S., Molla, M.M., \& Sarker, M.M.A. (2019). Numerical simulation of non-Newtonian power-law fluid flow in a lid-driven skewed cavity. International Journal of Applied and Computational Mathematics, 5(1), 14.

[17] Erturk, E., \& Dursun, B. (2007). Numerical solutions of 2-D steady incompressible flow in a driven skewed cavity. ZAMM-Journal of Applied Mathematics and Mechanics/Zeitschrift für Angewandte Mathematik und Mechanik: Applied Mathematics and Mechanics, 87(5), 377-392.

[18] Romano, F., \& Kuhlmann, H.C. (2017). Particle-boundary interaction in a shear-driven cavity flow. Theoretical and Computational Fluid Dynamics, 31(4), 427-445.

[19] de Vicente, J., Basley, J., Meseguer-Garrido, F., Soria, J., \& Theofilis, V. (2014). Three-dimensional instabilities over a rectangular open cavity: from linear stability analysis to experimentation. Journal of Fluid Mechanics, 748, 189-220.

[20] Jenson, V. (1959). Viscous flow round a sphere at low Reynolds numbers $(<40)$. Proceedings of the Royal Society of London. Series A. Mathematical and Physical Sciences, 249(1258), 346-366.

[21] Haese, P., \& Teubner, M. (2002). Heat exchange in an attic space. International Journal of Heat and Mass Transfer, 45(25), 4925-4936.

[22] Hoffmann, K.A., \& Chiang, S.T. (2000). Computational Fluid Dynamics. Vol. I. Engineering Education System.

[23] Tu, J., Yeoh, G.H., \& Liu, C. (2018). Computational Fluid Dynamics: A Practical Approach. Butterworth-Heinemann.

[24] Kamel, A.G., Haraz, E.H., \& Hanna, S.N. (2020). Numerical simulation of three-sided lid-driven square cavity. Engineering Reports, 2(4), e12151.

[25] Moffatt, H.K. (1964). Viscous and resistive eddies near a sharp corner. Journal of Fluid Mechanics, 18(1), 1-18. 